\title{
NOTE ON COAREA FORMULAE IN THE HEISENBERG
} GROUP

\author{
Valentino Magnani
}

\begin{abstract}
We show a first nontrivial example of coarea formula for vectorvalued Lipschitz maps defined on the three dimensional Heisenberg group. In this coarea formula, integration on level sets is performed with respect to the 2-dimensional spherical Hausdorff measure, built by the Carnot-Carathéodory distance. The standard jacobian is replaced by the so called "horizontal jacobian", corresponding to the jacobian of the Pansu differential of the Lipschitz map. Joining previous results, we achieve all possible coarea formulae for Lipschitz maps defined on the Heisenberg group.
\end{abstract}

\section{Introduction}

The study of sub-Riemannian Geometry is recently carried out in several areas of Mathematics, such as Differential Geometry, PDEs, Geometric Measure Theory, Sobolev spaces and Geometric Control Theory. An account on these developments can be found for instance in $[\mathbf{1}],[\mathbf{1 0}]$, $[\mathbf{1 1}]$ and $[\mathbf{1 7}]$.

Aim of this note is to show the first nontrivial example of coarea formula for vector-valued maps, whose domain is a noncommutative stratified group endowed with its natural sub-Riemannian structure. Coarea formulae for real-valued maps on stratified groups and the more general Carnot-Carathéodory spaces have been largely studied by several authors in different contexts, [8], [9], [15], [16], [18], [20], [21]. Most of these results hold for functions of bounded variation, where the notion of perimeter measure plays a central role. In our case this notion cannot be employed since level sets have codimension higher than one. Moreover, the choice of target may affect even the existence of nontrivial coarea formulae, [13]. As main result of this note we obtain the following

2000 Mathematics Subject Classification. 28A75 (22E25).

Key words. Coarea formula, Heisenberg group. 
coarea formula

$$
\int_{A} u(x) J_{H} f(x) d x=\int_{\mathbb{R}^{2}}\left(\int_{f^{-1}(t) \cap A} u(y) d \mathcal{S}_{\mathbb{H}^{3}}^{2}(y)\right) d t,
$$

where $u: A \longrightarrow[0,+\infty]$ is a measurable function, $A$ is a measurable subset of the Heisenberg group $\mathbb{H}^{3}$, and $f: A \longrightarrow \mathbb{R}^{2}$ is a Lipschitz function with respect to the Euclidean distance. Heisenberg group certainly is the simplest model of stratified group, $[\mathbf{2 4}]$. The "sub-Riemannian" features of (1) are the horizontal jacobian $J_{H} f$ and the spherical Hausdorff measure $\mathcal{S}_{\mathbb{H}^{3}}^{2}$ with respect to the Carnot-Carathéodory distance. The horizontal jacobian corresponds to the jacobian of the matrix representing the Pansu differential (Definition 2.1) and the Carnot-Carathéodory distance is the control distance associated to the horizontal distribution of $\mathbb{H}^{3}$ (Section 2). These two objects are strictly related, as formulae (13) and (14) show. The measure $\mathcal{S}_{\mathbb{H}^{3}}^{2}$ only detects the non-horizontal part of level sets and the choice of $J_{H} f$ surprisingly fits this property. Lipschitz functions with respect to the Euclidean distance are also Lipschitz with respect to the Carnot-Carathéodory distance, but the converse is not true. This naturally raises the question of extending (1) to Lipschitz maps with respect to the Carnot-Carathéodory distance of $\mathbb{H}^{3}$. The difficulty of this problem clearly appears in examples of Lipschitz maps with respect to the Carnot-Carathéodory distance which are nowhere differentiable on a set of full measure, [15]. Coarea formula (1) fits into the general coarea formula stated in [13], whose validity for arbitrary stratified groups is still an open problem. Nonetheless, formula (1) allows us to complete the picture of all possible coarea formulae for Lipschitz maps defined on $\mathbb{H}^{3}$, as we show in Theorem 5.2.

In ending, although our proof of coarea formula suggests a clear pattern for its extension to higher dimensional Heisenberg groups, a number of new difficulties appears in this case, as we explain in Remark 4.4. In this perspective, the present note becomes the first step to understand more general coarea formulae in higher dimensional stratified groups, where the intriguing geometry of higher codimensional sets is a new terrain for further investigations.

\section{A digest of basic notions}

We begin this section introducing the 3 -dimensional Heisenberg group. This is a simply connected Lie group $\mathbb{H}^{3}$ whose Lie algebra $\mathfrak{h}^{3}$ is endowed with a basis $\left(X_{1}, X_{2}\right)$ satisfying the nontrivial bracket relations $\left[X_{1}, X_{2}\right]=2 X_{3}$. We will identify the Lie algebra $\mathfrak{h}^{3}$ with the 
isomorphic Lie algebra of left invariant vector fields of $\mathbb{H}^{3}$. The exponential map exp: $\mathfrak{h}^{3} \longrightarrow \mathbb{H}^{3}$ is a diffeomorphism, then it is possible to introduce global coordinates on $\mathbb{H}^{3}$. We consider $F: \mathbb{R}^{3} \longrightarrow \mathbb{H}^{3}$ defined by

$$
F(x)=\exp \left(x_{1} X_{1}+x_{2} X_{2}+x_{3} X_{3}\right) .
$$

We will assume throughout that a system of coordinates defined by (2) is fixed. This allows us to identify $\mathbb{H}^{3}$ with $\mathbb{R}^{3}$. The vector fields $\left(X_{1}, X_{2}, X_{3}\right)$ with respect to our coordinates read as $X_{1}=\partial_{x_{1}}-x_{2} \partial_{x_{3}}, X_{2}=\partial_{x_{2}}+$ $x_{1} \partial_{x_{3}}$ and $X_{3}=\partial_{x_{3}}$. The group operation is represented by the formula

$$
x \cdot y=\left(x_{1}+y_{1}, x_{2}+y_{2}, x_{3}+y_{3}+x_{1} y_{2}-x_{2} y_{1}\right) .
$$

A natural family of dilations which respects the group operation (3) can be defined by setting $\delta_{r}(x)=\left(r x_{1}, r x_{2}, r^{2} x_{3}\right)$ for every $r>0$. In fact, the map $\delta_{r}: \mathbb{H}^{3} \longrightarrow \mathbb{H}^{3}$ defined above is a group homomorphism with respect to the operation (3). Our frame $\left(X_{1}, X_{2}, X_{3}\right)$ admits a dual basis $\left(d x_{1}, d x_{2}, \vartheta\right)$ of one-forms on $\mathbb{H}^{3}$, where the contact form $\vartheta$ can be explicitly written as

$$
\vartheta=d x_{3}+x_{2} d x_{1}-x_{1} d x_{2} .
$$

The vector fields $X_{1}, X_{2}$ span a smooth distribution of 2-dimensional planes, which define all horizontal directions of $\mathbb{H}^{3}$. A point $\gamma(t)$ of a differentiable curve $\gamma:[a, b] \longrightarrow \mathbb{H}^{3}$ is characteristic if $\gamma^{\prime}(t)$ is a horizontal direction and it is called transverse otherwise. Absolutely continuous curves which are a.e. characteristic are called horizontal curves, [1]. The sub-Riemannian metric structure of $\mathbb{H}^{3}$ is obtained fixing a left invariant Riemannian metric on $\mathbb{H}^{3}$ and defining the Carnot-Carathéodory distance between two points as the infimum over Riemannian lengths of horizontal curves joining these points. Vector fields $X_{1}$ and $X_{2}$ satisfy the Lie bracket generating condition, therefore the Chow theorem implies that every couple of points is joined by at least one horizontal curve, see for instance [1, p. 15]. As a result, the Carnot-Carathéodory distance is well defined.

Through coordinates (2) we can introduce the one dimensional Hausdorff measure $\mathcal{H}^{1}$ on $\mathbb{H}^{3}$ with respect to the Euclidean distance in $\mathbb{R}^{3}$. This measure clearly depends on our coordinates, however our final results will be formulated in intrinsic terms. We will assume throughout that Lipschitz functions on subsets of $\mathbb{H}^{3}$ are considered with respect to the Euclidean distance of $\mathbb{H}^{3}$. The symbol $|\cdot|$ will denote the Euclidean norm. By contrast with Analysis in Euclidean spaces, where the Euclidean distance is the most natural choice, in the Heisenberg group 
several distances have been introduced for different purposes. However, all of these distances are "homogeneous", namely, they are left invariant and satisfy the relation $\rho\left(\delta_{r} y, \delta_{r} z\right)=r \rho(y, z)$ for every $y, z \in \mathbb{H}^{3}$ and $r>0$. To simplify notations we write $\rho(x, 0)=\rho(x)$, where 0 denotes either the origin of $\mathbb{R}^{3}$ or the unit element of $\mathbb{H}^{3}$. The open ball of center $x$ and radius $r>0$ with respect to a homogeneous distance is denoted by $B_{x, r}$. The Carnot-Carathéodory distance is an important example of homogeneous distance. However, all of our computations hold for a general homogeneous distance, therefore in the sequel $\rho$ will denote a homogeneous distance, if not stated otherwise. Note that the Hausdorff dimension of $\mathbb{H}^{3}$ with respect to any homogeneous distance is four.

Before introducing the next definition we recall that any $L: \mathbb{H}^{3} \longrightarrow \mathbb{R}^{k}$ is a G-linear map if it is a group homomorphism satisfying the homogeneity property $L\left(\delta_{r} x\right)=r L(x)$ for every $x \in \mathbb{H}^{3}$ and $r>0$. Note that G-linear maps are also linear in the usual sense, as we identifiy $\mathbb{H}^{3}$ with $\mathbb{R}^{3}$.

G-linear maps constitute the family of intrinsic differentials, as we clarify in the following definition.

Definition 2.1 (P-differentiability). Let $f: \Omega \longrightarrow \mathbb{R}^{k}$, where $\Omega$ is an open subset of $\mathbb{H}^{3}$. We say that $f$ is $P$-differentiable at $x \in \Omega$ if there exists a G-linear map $L: \mathbb{H}^{3} \longrightarrow \mathbb{R}^{k}$ such that $\mid f(x \cdot h)-f(x)-$ $L(h) \mid \rho(h)^{-1} \longrightarrow 0$ as $\rho(h) \rightarrow 0$. The G-linear map $L$ with the previous property is uniquely defined and it is called the $P$-differential of $f$ at $x$. We use the notation $D f(x)$ to indicate the P-differential $L$.

The notion of P-differentiability has been introduced by Pansu in the more general framework of stratified groups, $[\mathbf{2 2}]$. One can check by direct computation that $f: \Omega \longrightarrow \mathbb{R}^{k}$ is P-differentiable at $x \in \Omega$ if it is differentiable at $x$ in the usual sense. Note that the converse is not true. The $k \times 3$ matrix representing $D f(x)$ can be written as follows

$$
D f(x)=\left[\begin{array}{ccc}
X_{1} f^{1}(x) & X_{2} f^{1}(x) & 0 \\
X_{1} f^{2}(x) & X_{2} f^{2}(x) & 0 \\
\vdots & \vdots & \vdots \\
X_{1} f^{k}(x) & X_{2} f^{k}(x) & 0
\end{array}\right] .
$$

We denote by $\nabla f(x)$ the $k \times 3$ matrix $\left(f_{x_{j}}^{i}\right)_{j=1,2,3}^{i=1, \ldots, k}$ representing the standard differential $d f(x)$ of $f$ at $x$. The horizontal jacobian $J_{H} f(x)$ of $f$ at $x$ is defined by taking the standard jacobian of the matrix (5). The standard jacobian of $f$ at $x$ is denoted by $J f(x)$. The Lebesuge measure 
of a measurable subset $A$ in $\mathbb{H}^{3}$ is denoted by $|A|$ and the $d$-dimensional spherical Hausdorff measure $\mathcal{S}^{d}$ is always considered with respect to the fixed homogeneous distance $\rho$. Note that our definition of spherical Hausdorff measure differs from the standard one of [6], in that the volume of the $d$-dimensional ball $\omega_{d}$ is replaced by one. The reason for this choice clearly appears in Corollary 3.2, where the "natural" dimensional constant $2 / \rho((0,0,1))^{2}$ in the definition of $\mathcal{S}_{\mathbb{H}^{3}}^{2}$ replaces $\omega_{1}=2$.

\section{Intrinsic measure of transverse curves}

The present section is devoted to the blow-up of $C^{1}$ curves with respect to a homogeneous distance. As a consequence, we achieve formula (13), corresponding to the integral representation of the 2-dimensional spherical Hausdorff measure of a transverse curve. This formula has been first obtained by Pansu, [20]. To make this note more selfcontained, here we recall its proof. In the sequel, $\Omega$ will denote an open subset of $\mathbb{H}^{3}$ and coordinates (2) will be understood, then we will identify $\Omega$ with an open subset of $\mathbb{R}^{3}$.

Theorem 3.1. Let $\gamma \subset \mathbb{H}^{3}$ be a one-dimensional immersed submanifold of class $C^{1}$ and let $x \in \gamma$. If $\gamma$ is transverse at $x$, then $\|\vartheta(x)\|>0$ and the limit

$$
\lim _{r \rightarrow 0^{+}} \frac{\mathcal{H}^{1}\left(\gamma \cap B_{x, r}\right)}{r^{2}}=\frac{c}{\|\vartheta(x)\|}
$$

holds, where $c=2 / \rho((0,0,1))^{2}$.

Proof: Let us denote by the same symbol $\gamma: J \longrightarrow \mathbb{H}^{3}$ a local parametrization of the immersed submanifold $\gamma$ near the point $x$, such that $\gamma(0)=x$ and $J$ is an open neighbourhood of zero. Defining the subset $I_{x, r}=$ $\{t \in J \mid \rho(\gamma(t), x)<r\}$, we have

$$
\mathcal{H}^{1}\left(\gamma \cap B_{x, r}\right)=\int_{I_{x, r}}\left|\gamma^{\prime}(t)\right| d t,
$$

then the change of variable $t=r^{2} \tau$ yields

$$
\frac{\mathcal{H}^{1}\left(\gamma \cap B_{x, r}\right)}{r^{2}}=\int_{r^{-2} I_{x, r}}\left|\gamma^{\prime}\left(r^{2} \tau\right)\right| d \tau,
$$

where we have defined $r^{-2} I_{x, r}=\left\{\tau \in r^{-2} J \mid \rho\left(\gamma\left(r^{2} \tau\right), x\right)<r\right\}$. The left invariance of $\rho$ and the homogeneity of dilations yield

$$
r^{-2} I_{x, r}=\left\{\tau \in r^{-2} J \mid \rho\left(\delta_{1 / r}\left(x^{-1} \gamma\left(r^{2} \tau\right)\right)\right)<1\right\} .
$$


The group law (3) allows us to compute the components of $\delta_{1 / r}\left(x^{-1} \gamma\left(r^{2} \tau\right)\right)$ in $\mathbb{R}^{3}$, obtaining

(8) $\left[\delta_{1 / r}\left(x^{-1} \gamma\left(r^{2} \tau\right)\right)\right]_{j}=\frac{\gamma_{j}\left(r^{2} \tau\right)-\gamma_{j}(0)}{r} \longrightarrow 0 \quad$ as $\quad r \rightarrow 0^{+}$,

for every $j=1,2$. Computing the third component we get

$$
\begin{aligned}
& {\left[\delta_{1 / r}\left(x^{-1} \gamma\left(r^{2} \tau\right)\right)\right]_{3}=\frac{\gamma_{3}\left(r^{2} \tau\right)-\gamma_{3}(0)-\gamma_{1}(0) \gamma_{2}\left(r^{2} \tau\right)+\gamma_{2}(0) \gamma_{1}\left(r^{2} \tau\right)}{r^{2}}} \\
& \quad=\frac{\gamma_{3}\left(r^{2} \tau\right)-\gamma_{3}(0)-\gamma_{1}(0)\left(\gamma_{2}\left(r^{2} \tau\right)-\gamma_{2}(0)\right)+\gamma_{2}(0)\left(\gamma_{1}\left(r^{2} \tau\right)-\gamma_{1}(0)\right)}{r^{2}}
\end{aligned}
$$

and from the expression of the contact form (4) we conclude that

(9) $\left[\delta_{1 / r}\left(x^{-1} \gamma\left(r^{2} \tau\right)\right)\right]_{3} \longrightarrow \tau\left(\gamma_{3}^{\prime}(0)-\gamma_{1}(0) \gamma_{2}^{\prime}(0)+\gamma_{2}(0) \gamma_{1}^{\prime}(0)\right)$

$$
=\tau \vartheta\left(\gamma(0), \gamma^{\prime}(0)\right) \text {. }
$$

By definition of contact form a vector $V \in T_{x} \mathbb{H}^{3}$ is horizontal if and only if $\vartheta(x, V)=0$, then $\vartheta\left(x, \gamma^{\prime}(0)\right) \neq 0$ and $\|\vartheta(x)\|>0$, because $\gamma$ is transverse at $x$. Limits (8) and (9) imply that for every $t \in \mathbb{R} \backslash\left\{\tau_{0},-\tau_{0}\right\}$ we have

$$
\mathbf{1}_{r^{-2} I_{x, r}}(t) \longrightarrow \mathbf{1}_{I_{x, 0}^{\prime}}(t) \quad \text { as } \quad r \rightarrow 0^{+}
$$

where $\tau_{0}=\left|\vartheta\left(x, \gamma^{\prime}(0)\right)\right|^{-1} \rho((0,0,1))^{-2}$ and

$$
I_{x, 0}^{\prime}=\left\{\tau \in \mathbb{R}|| \tau \vartheta\left(x, \gamma^{\prime}(0)\right) \mid \rho((0,0,1))^{2}<1\right\}=\left(-\tau_{0}, \tau_{0}\right) .
$$

Finally, formulae (7), (11) and limit (10) along with Lebesgue convergence theorem yield

$$
\frac{\mathcal{H}^{1}\left(\gamma \cap B_{x, r}\right)}{r^{2}} \longrightarrow 2 \tau_{0}\left|\gamma^{\prime}(0)\right| \quad \text { as } \quad r \rightarrow 0^{+} .
$$

This completes the proof.

Corollary 3.2 (Integral representation). Let $\gamma \subset \mathbb{H}^{3}$ be a one-dimensional immersed submanifold of class $C^{1}$ which is $\mathcal{S}^{2}$-a.e. transverse. Then we have the formula

$$
\mathcal{S}_{\mathbb{H}^{3}}^{2}(\gamma)=\int_{\gamma}\|\vartheta(x)\| d \mathcal{H}^{1}(x),
$$

where $c=2 / \rho((0,0,1))^{2}$ and $\mathcal{S}_{\mathbb{H}^{3}}^{2}=c \mathcal{S}^{2}$. 
Proof: It suffices to define the new measure $\mu=\|\vartheta(x)\| \mathcal{H}^{1}$, then Theorem 3.1 along with standard differentiability theorems applied to $\mu$, see for instance Theorem 2.10.17(2) and Theorem 2.10.18(1) of [6], lead us to our claim.

Remark 3.3. Note that formula (13) can be also expressed with respect to any left invariant metric $g$, replacing the role of the Euclidean distance. In fact, we have the equalities

$$
\begin{aligned}
\int_{\gamma}\|\vartheta(x)\|_{g} d \mathcal{H}_{g}^{1} & =\int_{J} \frac{\left|\vartheta\left(\gamma(t), \gamma^{\prime}(t)\right)\right|}{\left|\gamma^{\prime}(t)\right|_{g}}\left|\gamma^{\prime}(t)\right|_{g} d t \\
& =\int_{J} \frac{\left|\vartheta\left(\gamma(t), \gamma^{\prime}(t)\right)\right|}{\left|\gamma^{\prime}(t)\right|}\left|\gamma^{\prime}(t)\right| d t \\
& =\int_{\gamma}\|\vartheta(x)\| d \mathcal{H}^{1}=\mathcal{S}_{\mathbb{H}^{3}}^{2}(\gamma),
\end{aligned}
$$

where $\mathcal{H}_{g}^{1}$ is the one dimensional Hausdorff measure with respect to the Riemannian distance and $|\cdot|_{g}$ denotes the Riemannian norm. This remark emphasizes the auxiliary role of the Euclidean distance.

\section{Coarea formula for vector valued maps}

The purpose of this section is to prove our main result stated in Theorem 4.3. To do this, the next theorem constitutes the key tool.

Theorem 4.1. Let $f: \Omega \longrightarrow \mathbb{R}^{2}$ be a $C^{1}$ function, $x \in \Omega$ and assume that $d f(x): \mathbb{H}^{3} \longrightarrow \mathbb{R}^{2}$ is surjective. Then there exists a neighbourhood $U$ of $x$ such that for every $y$ belonging to the one-dimensional submanifold $f^{-1}(f(x)) \cap U$ we have

$$
J_{H} f(y)=\|\vartheta(y)\| J f(y) .
$$

Proof: We denote by $(\nabla f)_{i_{1} i_{2}}$ the $2 \times 2$ submatrix of $\nabla f$ with columns $i_{1}$ and $i_{2}$, and by $M_{i_{1} i_{2}}(\nabla f)$ the minor $\operatorname{det}\left((\nabla f)_{i_{1} i_{2}}\right)$. By hypothesis the matrix $\nabla f(x)$ has rank two, therefore we assume for instance that $M_{13}(\nabla f(x)) \neq 0$. The implicit function theorem yields a $C^{1}$ immersion $\gamma: J \longrightarrow \mathbb{H}^{3}$ such that $\gamma(0)=x$ and $f(\gamma(t))=f(x)$ for every $t$ belonging to the open interval $J$ containing the origin. In addition, the curve $\gamma$ can be represented as $\gamma(t)=\left(\gamma_{1}(t), t, \gamma_{3}(t)\right)$, where $\gamma_{j}: J \longrightarrow \mathbb{R}$ is a $C^{1}$ function for $j=1,2$. By a simple and elementary calculation, the differentation of equality $f\left(\left(\gamma_{1}(t), t, \gamma_{2}(t)\right)\right)=f(x)$ leads us to the 
formula

$$
\left[\begin{array}{c}
\gamma_{1}^{\prime} \\
\gamma_{3}^{\prime}
\end{array}\right]=-\frac{1}{M_{13}(\nabla f)}\left[\begin{array}{cc}
f_{x_{3}}^{2} & -f_{x_{3}}^{1} \\
-f_{x_{1}}^{2} & f_{x_{1}}^{1}
\end{array}\right]\left[\begin{array}{l}
f_{x_{2}}^{1} \\
f_{x_{2}}^{2}
\end{array}\right]
$$

where we have explicitly written the inverse matrix $\left((\nabla f)_{13}\right)^{-1}$. Expression (15) yields

$$
\gamma_{1}^{\prime}=-\frac{M_{23}(\nabla f)}{M_{13}(\nabla f)} \quad \text { and } \quad \gamma_{3}^{\prime}=-\frac{M_{12}(\nabla f)}{M_{13}(\nabla f)} .
$$

Using the definition of $J_{H} f$ and the explicit expressions of operators $X_{j}$ one can achieve the following equality

$$
J_{H} f(x)=\left|M_{12}(\nabla f(x))+x_{1} M_{13}(\nabla f(x))-x_{2} M_{32}(\nabla f(x))\right| .
$$

As a consequence of this formula, dividing both terms of the quotient $J_{H} f / J f$ by $\left|M_{13}(\nabla f)\right|$ and using (16), we obtain

(18) $\frac{J_{H} f(\gamma(t))}{J f(\gamma(t))}=\frac{\left|\gamma_{3}^{\prime}(t)-\gamma_{1}(t)+t \gamma_{1}^{\prime}(t)\right|}{\left|\gamma^{\prime}(t)\right|}=\frac{\left|\vartheta\left(\gamma(t), \gamma^{\prime}(t)\right)\right|}{\left|\gamma^{\prime}(t)\right|}=\|\vartheta(\gamma(t))\|$.

Clearly, either possible cases $M_{12}(\nabla f(x)) \neq 0$ or $M_{23}(\nabla f(x)) \neq 0$ would lead us to the same formula, due to its intrinsic form.

Remark 4.2. Note that in the statement of the next theorem the horizontal jacobian $J_{H} f$ is considered when $f$ is defined on a measurale set instead of an open set. This refers to a slightly more general notion of P-differentiability, where interior points of the domain $A$ are replaced with density points. Even in this case the P-differential is uniquely defined, see Definition 7 and Proposition 2.2 of [12] for more details.

Theorem 4.3 (Coarea formula). Let $f: A \longrightarrow \mathbb{R}^{2}$ be a Lipschitz map, where $A \subset \mathbb{H}^{3}$ is a measurable subset. Then for every measurable function $u: A \longrightarrow[0,+\infty]$ the formula

$$
\int_{A} u(x) J_{H} f(x) d x=\int_{\mathbb{R}^{2}}\left(\int_{f^{-1}(t) \cap A} u(y) d \mathcal{S}_{\mathbb{H}^{3}}^{2}(y)\right) d t
$$

holds, where $c=2 / \rho((0,0,1))^{2}$ and $\mathcal{S}_{\mathbb{H}^{3}}^{2}=c \mathcal{S}^{2}$.

Proof: We first prove (19) in the case $f$ is defined on all of $\mathbb{H}^{3}$ and is of class $C^{1}$. Let $\Omega$ be an open subset of $\mathbb{H}^{3}$. In view of the Euclidean coarea formula we have

$$
\int_{\Omega} u(x) J f(x) d x=\int_{\mathbb{R}^{2}}\left(\int_{f^{-1}(t) \cap \Omega} u(y) d \mathcal{H}^{1}(y)\right) d t
$$


where $u: \Omega \longrightarrow[0,+\infty]$ is a measurable function, see for instance $[\mathbf{6}]$. Now we define $u(x)=J_{H} f(x) \mathbf{1}_{\{J f \neq 0\} \cap \Omega}(x) / J f(x)$ and use (20), obtaining

(21) $\int_{\Omega} J_{H} f(x) d x=\int_{\mathbb{R}^{2}}\left(\int_{f^{-1}(t) \cap \Omega} \frac{J_{H} f(x) \mathbf{1}_{\{J f \neq 0\}}(x)}{J f(x)} d \mathcal{H}^{1}(y)\right) d t$.

The validity of (20) also implies that for a.e. $t \in \mathbb{R}^{2}$ the set of points of $f^{-1}(t)$ where $J f$ vanishes is $\mathcal{H}^{1}$-negligible, then the previous formula becomes

$$
\int_{\Omega} J_{H} f(x) d x=\int_{\mathbb{R}^{2}}\left(\int_{f^{-1}(t) \cap \Omega} \frac{J_{H} f(x)}{J f(x)} d \mathcal{H}^{1}(y)\right) d t .
$$

By Theorem 2.7 of $[\mathbf{1 3}]$, for a.e. $t \in \mathbb{R}^{2}$ we have that $\mathcal{S}^{2}\left(C_{t} \cap \Omega\right)=0$, where we have defined

$$
C_{t}=\left\{y \in f^{-1}(t) \cap \Omega \mid J_{H} f(y)=0\right\} .
$$

As a result, from formulae (13) and (14) we have proved that for a.e. $t \in \mathbb{R}^{2}$ the equalities

$$
\int_{f^{-1}(t) \cap \Omega} \frac{J_{H} f(x)}{J f(x)} d \mathcal{H}^{1}(y)=\mathcal{S}_{\mathbb{H}^{3}}^{2}\left(f^{-1}(t) \cap \Omega \backslash C_{t}\right)=\mathcal{S}_{\mathbb{H}^{3}}^{2}\left(f^{-1}(t) \cap \Omega\right)
$$

hold, therefore we have achieved

$$
\int_{\Omega} J_{H} f(x) d x=\int_{\mathbb{R}^{2}} \mathcal{S}_{\mathbb{H}^{3}}^{2}\left(f^{-1}(t) \cap \Omega\right) d t
$$

The arbitrary choice of $\Omega$ yields the validity of (23) also for arbitrary closed sets. Then, approximation of measurable sets by closed ones, Borel regularity of $\mathcal{S}_{\mathbb{H}^{3}}^{2}$ and the coarea estimate 2.10 .25 of $[\mathbf{6}]$ extend the validity of (23) to the following one

$$
\int_{A} J_{H} f(x) d x=\int_{\mathbb{R}^{2}} \mathcal{S}_{\mathbb{H}^{3}}^{2}\left(f^{-1}(t) \cap A\right) d t
$$

where $A$ is a measurable subset of $\mathbb{H}^{3}$. Now we consider the general case, where $f: A \longrightarrow \mathbb{R}^{2}$ is a Lipschitz map defined on a measurable bounded subset $A$ of $\mathbb{H}^{3}$. Let $f_{1}: \mathbb{H}^{3} \longrightarrow \mathbb{R}^{2}$ be a Lipschitz extension of $f$, namely, $f_{1 \mid A}=f$ holds. Due to the Whitney extension theorem (see for instance 3.1 .15 of $[\mathbf{6}]$ ) for every arbitrarily fixed $\varepsilon>0$ there exists a $C^{1}$ function $f_{2}: \mathbb{H}^{3} \longrightarrow \mathbb{R}^{2}$ such that the open subset $O=$ $\left\{z \in \mathbb{H}^{3} \mid f_{1}(z) \neq f_{2}(z)\right\}$ has Lebesgue measure less than or equal to $\varepsilon$. The map $f$ is a.e. differentiable in the Euclidean sense, then it is also 
a.e. P-differentiable. As we have mentioned in Remark 4.2 the horizontal jacobian $J_{H} f$ is well defined and we can consider the estimate

$$
\begin{aligned}
\mid \int_{A} J_{H} f(x) d x & -\int_{\mathbb{R}^{2}} \mathcal{S}_{\mathbb{H}^{3}}^{2}\left(f^{-1}(t) \cap A\right) d t \mid \\
& \leq \int_{A \cap O} J_{H} f(x) d x+\int_{\mathbb{R}^{2}} \mathcal{S}_{\mathbb{H}^{3}}^{2}\left(f^{-1}(t) \cap A \cap O\right) d t .
\end{aligned}
$$

In fact, due to the first part of this proof, the following coarea formula for $C^{1}$ smooth maps holds

$$
\int_{A \backslash O} J_{H} f_{2}(x) d x=\int_{\mathbb{R}^{2}} \mathcal{S}_{\mathbb{H}^{3}}^{2}\left(f_{2}^{-1}(t) \cap A \backslash O\right) d t .
$$

Moreover, the equality $f_{2 \mid A \backslash O}=f_{\mid A \backslash O}$ implies that $J_{H} f_{2}=J_{H} f$ a.e. on $A \backslash O$, therefore

$$
\int_{A \backslash O} J_{H} f(x) d x=\int_{\mathbb{R}^{2}} \mathcal{S}_{\mathbb{H}^{3}}^{2}\left(f^{-1}(t) \cap A \backslash O\right) d t
$$

holds and inequality (25) is proved. Now we recall that the Euclidean distance can be estimated from above by any fixed homogeneous distance. Let $\rho_{g}$ denote the Riemannian distance defined by the left invariant metric $g$ fixed on $\mathbb{H}^{3}$. Then we have $\rho_{g} \leq \rho_{C C}$, where $\rho_{C C}$ is the Carnot-Carathéodory distance associated to $g$. The fact that the Riemannian distance is locally equivalent to the Euclidean distance and that the Carnot-Carathéodory distance is equivalent to any homogeneous distance prove our claim. As a consequence, due to the boundedness of $A$, the map $f$ is Lipschitz even with respect to the homogeneous distance $\rho$. Let us denote by $\operatorname{Lip}(f)$ the Lipschitz constant of $f$ with respect to the homogeneous distance $\rho$. Then there exists a constant $c_{0}$ depending on $\rho$ such that

$$
\|D f(x)\| \leq c_{0} \operatorname{Lip}(f)
$$

for a.e. $x \in A$. Then the algebraic inequality

$$
J_{H} f(x) \leq \sqrt{\left(X_{1} f^{1}(x)\right)^{2}+\left(X_{2} f^{1}(x)\right)^{2}} \sqrt{\left(X_{1} f^{2}(x)\right)^{2}+\left(X_{2} f^{2}(x)\right)^{2}}
$$

and (26) imply

$$
J_{H} f(x) \leq c_{0}^{2} \operatorname{Lip}(f)^{2}
$$

for a.e. $x \in A$. By virtue of the general coarea inequality 2.10 .25 of $[6]$ there exists a dimensional constant $c_{1}>0$ such that

$$
\int_{\mathbb{R}^{2}} \mathcal{S}_{\mathbb{H}^{3}}^{2}\left(f^{-1}(t) \cap A \cap O\right) d t \leq c_{1} \operatorname{Lip}(f)^{2} \mathcal{H}^{4}(O) .
$$


The fact that the 4-dimensional Hausdorff meaure $\mathcal{H}^{4}$ with respect to the homogeneous distance $\rho$ is proportional to the Lebesgue measure, gives us a constant $c_{2}>0$ such that

$$
\int_{\mathbb{R}^{2}} \mathcal{S}_{\mathbb{H}^{3}}^{2}\left(f^{-1}(t) \cap A \cap O\right) d t \leq c_{2} \operatorname{Lip}(f)^{2}|O| \leq c_{2} \operatorname{Lip}(f)^{2} \varepsilon .
$$

Thus, estimates (27) and (29) joined with inequality (25) yield

$$
\left|\int_{A} J_{H} f(x) d x-\int_{\mathbb{R}^{2}} \mathcal{S}_{\mathbb{H}^{3}}^{2}\left(f^{-1}(t) \cap A\right) d t\right| \leq\left(c_{0}^{2}+c_{2}\right) \operatorname{Lip}(f)^{2} \varepsilon .
$$

Letting $\varepsilon \rightarrow 0^{+}$, we have proved that

$$
\int_{A} J_{H} f(x) d x=\int_{\mathbb{R}^{2}} \mathcal{S}_{\mathbb{H}^{3}}^{2}\left(f^{-1}(t) \cap A\right) d t
$$

Finally, utilizing increasing sequences of step functions pointwise converging to $u$ and applying Beppo Levi convergence theorem, the proof of (19) is achieved in the case $A$ is bounded. If $A$ is not bounded, then one can take the limit of (19) where $A$ is replaced by $A_{k}$ and $\left\{A_{k}\right\}$ is an increasing sequence of measurable bounded sets whose union yields $A$. Then the Beppo Levi convergence theorem concludes the proof.

Remark 4.4. The proof of Theorem 4.3 suggests a method for its extension to higher dimensional Heisenberg groups. Applying this method two main problems appear. The first one is to reach an intrinsic characterization of the quotient $J_{H} f / J f$ in terms of the contact form and of possible new left invariant forms. The second one is the characterization of the blow-up limit in terms of these forms.

\section{All coarea formulae in the Heisenberg group}

This section collects all known coarea formulae for maps defined on the three dimensional Heisenberg group. We first recall the notion of coarea factor, see $[\mathbf{1 3}]$ for more information.

Definition 5.1 (Coarea factor). Let $L: \mathbb{H}^{3} \longrightarrow \mathbb{R}^{k}$ be a G-linear map, with $k \leq 4$. The coarea factor of $L$ with respect to the spherical Hausdorff measure $\mathcal{S}_{c}^{4-k}$ is the unique number $C_{k}(L)$ satisfying the relation

$$
C_{k}(L)|A|=\int_{\mathbb{R}^{k}} \mathcal{S}_{c}^{4-k}\left(L^{-1}(y) \cap A\right) d y
$$

for every measurable subset $A \subset \mathbb{H}^{3}$, where $c>0$ and $\mathcal{S}_{c}^{4-k}=c \mathcal{S}^{4-k}$. 
One can verify that the number $C_{k}(L) \geq 0$ is positive if and only if $L$ is surjective, see Proposition 1.12 of [13]. The general notion of coarea factor allows us to state in a unified way the validity of a family of coarea formulae, as we show in the following theorem.

Theorem 5.2. Let $A \subset \mathbb{H}^{3}$ be a measurable set and let $f: A \longrightarrow \mathbb{R}^{k}$ be a Lipschitz map with $1 \leq k \leq 4$. Let $\rho$ be the Carnot-Carathéodory distance. Then for any measurable function $u: A \longrightarrow[0,+\infty]$ the following equality

$$
\int_{A} u(x) C_{k}(D f(x)) d x=\int_{\mathbb{R}^{k}} \int_{f^{-1}(t) \cap A} u(z) d \mathcal{S}_{\mathbb{H}^{3}}^{4-k}(z) d t
$$

holds and it becomes the trivial identity $0=0$ if $k=3,4$. The formulae $\mathcal{S}_{\mathbb{H}^{3}}^{3}=\alpha \mathcal{S}_{\rho}^{3}$ and $\mathcal{S}_{\mathbb{H}^{3}}^{2}=2 \rho((0,0,1))^{-2} \mathcal{S}_{\rho}^{2}$ hold, where $\alpha$ is the metric factor of the Carnot-Carathéodory distance and $\mathcal{S}_{\rho}^{d}$ is the d-dimensional spherical Hausdorff measure built by the distance $\rho$. The coarea factor $C_{k}(D f(x))$ is considered with respect to $\mathcal{S}_{\mathbb{H}^{3}}^{4-k}$.

In the case $k=1$, Theorem 5.2 was first proved by Pansu, [20], [21], where

$$
C_{1}(D f(x))=\sqrt{X_{1} f(x)^{2}+X_{2} f(x)^{2}} .
$$

The coarea factor $\alpha$ in the definition of $\mathcal{S}_{\mathbb{H}^{3}}^{3}$ has been introduced in [14], where (32) has been extended to real-valued Lipschitz maps on stratified groups. Here the metric factor is constant due to the invariant property of the Carnot-Carathéodory distance with respect to horizontal isometries, see $[\mathbf{1 4}]$ for more information. In the case $k=2$, the validity of (19) for any G-linear map and the definition of coarea factor easily imply the equality

$$
C_{2}(D f(x))=J_{H} f(x),
$$

then Theorem 5.2 is a consequence of Theorem 4.3. If $k=3,4$, then the general coarea inequality of $[\mathbf{1 3}]$ can be applied. In fact, any G-linear map $L: \mathbb{H}^{3} \longrightarrow \mathbb{R}^{k}$ cannot be surjective in this case, as it easily follows from its matrix representation (5). Then the number $C_{k}(D f(x))$ is always vanishing and the general coarea inequality of [13] yields the trivial identity $0=0$. The same argument applies to stratified groups $\mathbb{M}$ in the target, having topological dimension greater than or equal to 4 , see also Subsection 2.1 of [13]. The only possible noncommutative stratified group in the target giving a nontrivial coarea formula is the three dimensional Heisenberg group itself. In this case the coarea formula coincides with the area formula, [12], and the map $f$ is assumed to be Lipschitz with respect to the homogeneous distance of $\mathbb{H}^{3}$. 


\section{References}

[1] A. BellaïChe And J.-J. Risler, EDS., "Sub-Riemannian geometry", Progress in Mathematics 144, Birkhäuser Verlag, Basel, 1996.

[2] A.-P. CALderón AND A. ZyGmund, Local properties of solutions of elliptic partial differential equations, Studia Math. 20 (1961), $171-225$.

[3] L. Capogna, D. Danielli and N. Garofalo, The geometric Sobolev embedding for vector fields and the isoperimetric inequality, Comm. Anal. Geom. 2(2) (1994), 203-215.

[4] L. J. Corwin and F. P. Greenleaf, "Representations of nilpotent Lie groups and their applications. Part I. Basic theory and examples", Cambridge Studies in Advanced Mathematics 18, Cambridge University Press, Cambridge, 1990.

[5] E. Falbel and F. Jean, Measures of transverse paths in subRiemannian geometry, J. Anal. Math. 91 (2003), 231-246.

[6] H. Federer, "Geometric measure theory", Die Grundlehren der mathematischen Wissenschaften 153, Springer-Verlag New York Inc., New York, 1969.

[7] G. B. Folland And E. M. Stein, "Hardy spaces on homogeneous groups", Mathematical Notes 28, Princeton University Press, Princeton, N.J.; University of Tokyo Press, Tokyo, 1982.

[8] B. Franchi, R. Serapioni and F. Serra Cassano, MeyersSerrin type theorems and relaxation of variational integrals depending on vector fields, Houston J. Math. 22(4) (1996), 859-890.

[9] N. Garofalo and D.-M. Nhieu, Isoperimetric and Sobolev inequalities for Carnot-Carathéodory spaces and the existence of minimal surfaces, Comm. Pure Appl. Math. 49(10) (1996), 1081-1144.

[10] P. Hajeasz and P. Koskela, Sobolev met Poincaré, Mem. Amer. Math. Soc. 145(688) (2000), 101 pp.

[11] I. Kupka, Géométrie sous-riemannienne, Séminaire Bourbaki, Vol. 1995/96, Astérisque 241 (1997), Exp. No. 817, 5, 351-380.

[12] V. Magnani, Differentiability and area formula on stratified Lie groups, Houston J. Math. 27(2) (2001), 297-323.

[13] V. Magnani, On a general coarea inequality and applications, Ann. Acad. Sci. Fenn. Math. 27(1) (2002), 121-140.

[14] V. Magnani, A blow-up theorem for regular hypersurfaces on nilpotent groups, Manuscripta Math. 110(1) (2003), 55-76.

[15] V. Magnani, The coarea formula for real-valued Lipschitz maps on stratified groups, Math. Nachr. (to appear). 
[16] M. Miranda, JR., Functions of bounded variation on "good" metric spaces, J. Math. Pures Appl. (9) 82(8) (2003), 975-1004.

[17] R. Montgomery, "A tour of subriemannian geometries, their geodesics and applications", Mathematical Surveys and Monographs 91, American Mathematical Society, Providence, RI, 2002.

[18] R. Monti and F. Serra Cassano, Surface measures in CarnotCarathéodory spaces, Calc. Var. Partial Differential Equations 13(3) (2001), 339-376.

[19] A. Nagel, E. M. Stein And S. Wainger, Balls and metrics defined by vector fields. I. Basic properties, Acta Math. 155(1-2) (1985), 103-147.

[20] P. Pansu, Géométrie du groupe de Heisenberg, Thèse de 3ème cycle, Université Paris 7 (1982).

[21] P. PAnsu, Une inégalité isopérimétrique sur le groupe de Heisenberg, C. R. Acad. Sci. Paris Sér. I Math. 295(2) (1982), 127-130.

[22] P. Pansu, Métriques de Carnot-Carathéodory et quasiisométries des espaces symétriques de rang un, Ann. of Math. (2) 129(1) (1989), 1-60.

[23] S. Semmes, On the nonexistence of bi-Lipschitz parameterizations and geometric problems about $A_{\infty}$-weights, Rev. Mat. Iberoamericana 12(2) (1996), 337-410.

[24] E. M. STEIn, "Harmonic analysis: real-variable methods, orthogonality, and oscillatory integrals", Princeton Mathematical Series 43, Monographs in Harmonic Analysis III, Princeton University Press, Princeton, NJ, 1993.

Dipartimento di Matematica

via Buonarroti n. 2

56127 Pisa

Italy

E-mail address: magnani@dm.unipi.it

Primera versió rebuda el 18 de novembre de 2003, darrera versió rebuda el 7 de juny de 2004 . 